\title{
The Modulation of DNA Content: Proximate Causes and Ultimate Consequences
}

\author{
T. Ryan Gregory ${ }^{1}$ and Paul D.N. Hebert \\ Department of Zoology, University of Guelph, Guelph, Ontario N1G 2W1, Canada
}

\begin{abstract}
The forces responsible for modulating the large-scale features of the genome remain one of the most difficult issues confronting evolutionary biology. Although diversity in chromosomal architecture, nucleotide composition, and genome size has been well documented, there is little understanding of either the evolutionary origins or impact of much of this variation. The 80,000 -fold divergence in genome sizes among eukaryotes represents perhaps the greatest challenge for genomic holists. Although some researchers continue to characterize much variation in genome size as a mere by-product of an intragenomic selfish DNA "free-for-all" there is increasing evidence for the primacy of selection in molding genome sizes via impacts on cell size and division rates. Moreover, processes inducing quantum or doubling series variation in gametic or somatic genome sizes are common. These abrupt shifts have broad effects on phenotypic attributes at both cellular and organismal levels and may play an important role in explaining episodes of rapid-or even saltationalcharacter state evolution.
\end{abstract}

The advent of molecular tools such as PCR and automated sequencing has uncovered a veritable gold mine of information regarding the structures and functions of individual genes and gene complexes. However, as intellectually profitable as this "gene rush" has been, it has had the unfortunate consequence of leading many genetic prospectors to ignore what Maynard Smith (1982) has called "one of the most difficult, perhaps the most difficult, question in evolutionary biology"- the evolution of the large-scale features of the genome. In addition, it has led to a sometimes fierce dichotomy between geneticists focusing on the microevolutionary fates of individual genes and paleontologists concerned with interpreting the macroevolutionary patterns depicted in the fossil record. The gap between these two schools of thought is widened by the growing emphasis on patterns of punctuated change reported by the latter and the lack of a mechanistic explanation for them that is compatible with the wellestablished principles of population genetics demanded by the former.

It is increasingly recognized that measures of genetic divergence in random segments of the genome are unlikely to yield deep insights concerning the processes responsible for major morphological reconfigurations. As such, the key genes in developmental pathways or those that regulate the behavior of entire genomes represent more logical targets for analysis. The study of genomic properties is a particularly intriguing area for research because there is strong evidence that bulk DNA has important phenotypic effects and that several processes may lead to quantum variation in ge-

'Corresponding author.

E-MAIL rgregory@uoguelph.ca; FAX (519) 767-1656. nome size. This review explores the nature of variation in DNA content and investigates its role in provoking morphological—and evolutionary-change.

\section{The Nature of DNA Content Variation}

The basal genome size of an organism, also known as its C-value, is defined as the content of DNA (measured by weight or number of base pairs) in a single copy of the entire sequence of DNA found within cells of that organism. Basal genome sizes among prokaryotes vary only slightly, with those of Archea and Eubacteria ranging only an order of magnitude, from 0.5 to $5 \mathrm{Mb}$ (Krawiec and Riley 1990). The genome sizes of eukaryotes, on the other hand, vary $>80,000$-fold (Li 1997). Even among animals there is a nearly 3000 -fold variation, and in plants basal genomes sizes vary by a factor of $>6000$ (Li 1997). The question of how-and whygenome sizes have come to vary by such a surprising degree through evolutionary time is an important issue.

\section{Patterns of Genome Size Variation}

As with any other macroevolutionary topic, it is critical to ascertain whether the large changes in genome size now observed among taxa arose via the gradual accumulation or deletion of small segments of DNA or whether a more punctuated pattern of change predominates. In the case of birds, mammals, and teleost fish, where there is little variation in basal genome size, alterations in C-value ordinarily appear to have resulted from the gradual accretion and/or deletion of small blocks of DNA, as evidenced by the approximately normal distribution of genome sizes in these groups (Bachmann 1972; Bachmann et al. 1972; Gold et al. 1992). 
Because a convenient supply of nucleated blood cells is unavailable in invertebrates, information on genome size distributions in these organisms is less abundant than for vertebrates. However, it is clear that the patterns of genome size variation among single taxonomic groups of invertebrates often differ from those typical of vertebrates. Not only is variation in genome size among closely allied species often large, but Cvalues often vary in a discontinuous fashion despite stable chromosome numbers. For example, a recent survey of 79 species of polychaete worms showed a $>70$-fold variation (from 0.1 to $7.2 \mathrm{pg}$ ), with the genome sizes of closely allied species clearly varying in a quantum fashion (Sella et al. 1993; Gambi et al. 1997). Moreover, a comparison of genome sizes in different families of these organisms reveals the recurrence of specific nodal values, suggesting that there is a simple process enabling major shifts in C-value. This pattern of quantum variation in genome sizes is common among other invertebrates, particularly those showing high taxonomic diversity, such as insects and crustaceans. Similar patterns of genome size variation have, for example, been documented among copepods (McLaren et al. 1989), tardigrades (Garanga et al. 1996), anemones (Rothfels et al. 1966), aphids (Finston et al. 1995), and other insects (Hughes-Schrader and Schrader 1956), as well as among some plants (Rees 1972; Narayan 1982, 1983) and prokaryotes (Wallace and Morowitz 1973; Li 1997).

\section{Quantum Shifts in Genome Size}

The best-known mode of discontinuous change in DNA content is polyploidy. This process, which involves the multiplication of an entire chromosome set, is exceedingly common among plants (nearly 50\% of angiosperms are polyploid; Grant 1981). In those few cases in which discontinuous variation in DNA content is detected in vertebrates, such as among some fish and certain amphibians, cytogenetic studies confirm that it has derived from past polyploidization. Polyploidization also occurs in invertebrates but most quantum shifts in genome size in these organisms have apparently not resulted from this process, as species exhibiting different C-values share similar chromosome complements.

That genome sizes often do not vary continuously among species has been known for $>20$ years. In 1976, Sparrow and Naumann argued that the genome sizes of a whole range of organisms followed what they believed to be a doubling series. They found no association between genome size and chromosome number, making it clear that this doubling series was not a consequence of polyploidy. This process of genome doubling was termed cryptopolyploidy, and although no satisfactory mechanisms have been offered to explain it, two mutually compatible possibilities exist. First, this pattern of genome size modulation may result from the coordinate replication of all repetitive elements in the genome-a prospect supported by the discovery of mutations in the insect Chironomus that provoke the orchestrated replication of scattered genomic elements (Keyl 1965). Second, quantum shifts in C-value may simply occur via the additional replication of individual chromosomes. The accepted view that chromosomes possess a unineme structure appears to preclude this possibility, but the work supporting this view focused almost exclusively on vertebrates, which do not show cryptopolyploidy, and on other eukaryotes such as Drosophila and yeast, which have extremely small genomes.

\section{Gradual Shifts in Genome Size}

In some cases, such as among most vertebrates and to a certain degree among invertebrates, genome sizes vary in a more continuous fashion. These more gradual alterations of genome size are often attributed entirely to modulation of the repetitive DNA content of the genome. In fact, over a 25 -fold range in genome sizes relevant to most fish, reptiles, birds, and mammals (from 0.2 to $5 \mathrm{pg}$ ), the percentage of single-copy DNA shows no significant change (Fig. 1), indicating that within this range the amount of single-copy DNA shifts in concert with changes in genome size. Thus, the traditional claim that changes in single-copy DNA have little to do with genome size shifts seems exaggerated. The role of single-copy DNA in genome size modulation has been ignored in part because it has never been obvious how such radical shifts in the abundance of nonrepetitive DNA could arise.

The coding genes of eukaryotes ordinarily display an intron-exon structure whereby sequences of coding

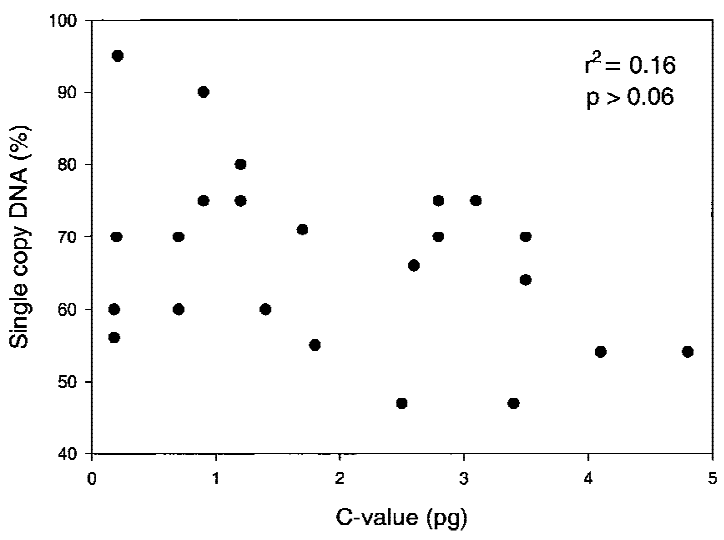

Figure 1 Relationship between haploid genome size and percent single copy DNA over a 25 -fold range in genome sizes among a wide range of animals (both vertebrates and invertebrates). Although very large genomes consist primarily of repetitive elements, there is little variation in the proportion of the genome made up of single copy sequences over this smaller range (which is relevant to most fish, reptiles, birds, and mammals). Data from John and Miklos (1988). 
DNA are interrupted by often lengthy stretches of seemingly superfluous nucleotides. Although the introns are excised during transcription and generally seem to play no critical role in gene function, their positions are remarkably conserved (Kersanach et al. 1994). The lengths of exons average $200 \mathrm{bp}$ in a broad range of eukaryotes (Hawkins 1988), but mean intron lengths vary $>20$-fold from a low of $25 \mathrm{bp}$ in Paramecium (Russell et al. 1994) to 60 bp in Drosophila and 650 bp in most vertebrates (Hawkins 1988). Given their regular interruptions with introns, a gene containing $1 \mathrm{~kb}$ of coding sequence would have a total length of $1.1 \mathrm{~kb}$ in Paramecium, $1.2 \mathrm{~kb}$ in Drosophila, and $3.6 \mathrm{~kb}$ in vertebrates. These differences suggest that variation in the amount of single-copy DNA among species may largely be explained by shifts in intron length. Moriyama et al. (1998) have suggested that differences in genome size among Drosophila species can be explained in part by differences in the lengths of their introns. Moreover, the positive correlation between genome size and bulk amounts of single-copy DNA suggests that the processes that impact intron length are also involved in regulating the size of other noncoding segments of the genome. Although differences in intron lengths may explain only a portion of the variation in genome size among organisms, they may provide a useful sentinel system by which to gauge the presence of factors causing the expansion or pruning of noncoding DNA on a genomewide scale. The precision with which such manipulations of DNA content appear to be carried out raises interesting questions regarding the nature of the forces and mechanisms responsible (Petrov and Hartl 1997). Some authors have proposed that the extremely small selection coefficients relating to additions or deletions of individual nucleotides could be sufficient, given enough time, to account for the gradual alterations in genome size observed in some groups (Charlesworth 1996). However, there is now increasing evidence that both shifts in intron lengths and in the amounts of other noncoding DNA are driven by genome-wide alterations in the incidence of deletion and insertion events, resulting in selection coefficients of a much more reasonable magnitude (Petrov et al. 1996).

\section{The C-Value Paradox}

The lack of a correlation between genome size and organismal complexity has so surprised biologists that it has come to be known as the "C-value paradox" (Thomas 1971). For example, Homo sapiens has a genome size 200 times smaller than that of Amoeba dubia (Li 1997). Moreover, it has been well established that the genomes of most eukaryotes contain thousands of times more DNA than required to carry out all necessary protein coding and regulatory functions. Some early attempts to explain this lack of an association between C-value and complexity proposed that the superfluous DNA present in large genomes acted as a storehouse of genetic variability that could be recruited by evolution should the need arise (Jain 1980). The fallacy of ascribing such foresight to the evolutionary process is now well recognized, but several plausible solutions to this puzzle remain.

\section{Junk, Parasites, Nucleotypes, and Nucleoskeletons}

Perhaps the simplest explanation for the C-value paradox offered to date is that the noncoding DNA is simply useless "junk" that accumulates over evolutionary time (e.g., Ohno 1972; Pagel and Johnstone 1992). This accumulation, it is argued, is driven by genetic drift, by mutation pressure resulting in duplications of genomic segments, or by the maintenance of "extinct" genes whose function has been lost. Thus, proponents of the junk DNA view suggest that there is a consistent drive unrelated to natural selection for increased genome size through time. The evidence supporting this view is weak; there is, for example, no evidence that "primitive" species invariably contain less DNA than their derived counterparts (coelacanths may have small genomes, but lungfish do not). In addition, it does not provide an explanation for the reductions in genome size that are known to have occurred in many lineages.

The characterization of the evolutionary process as the product of the differential reproductive success of individual (or groups of) selfish replicators (Dawkins 1976) led to the hypothesis that similar types of selection could operate on selfish elements acting to increase their representation within the genome, regardless of their effects on the "host" organism (Doolittle and Sapienza 1980; Orgel and Crick 1980). Under this view, the expansion or deletion of noncoding DNA represents the outcome of a continuous struggle between selfish DNA and the host genome, the former selected intragenomically to increase in number, and the latter selected to minimize the costs of replication associated with carrying a large amount of unnecessary genetic baggage. Thus, both the junk and selfish DNA hypotheses postulate that the C-value of a species is merely a by-product of the persistent accumulation of phenotypically neutral DNA that is excised only when it becomes too costly. Under these views, DNA content is not subject to direct adaptive modulation because it has no effects on the organismal phenotype.

The simple lack of a correlation between C-value and complexity is hardly strong grounds for concluding the phenotypic irrelevance of DNA content. It has been known for some time that the DNA content of a nucleus is closely related to the volume of its cell in a wide range of organisms (Mirsky and Ris 1951). Indeed, it has been asserted that "the most reliably established fact about genome evolution is that C-values are generally positively correlated with cell and nuclear vol- 
umes" (Cavalier-Smith 1982). This relationship with cell volume, in turn, results in strong inverse correlations between genome size and important life history characters such as rates of cell division (Cavalier-Smith 1985a), metabolism (Vinogradov 1995, 1997), and development (Sessions and Larson 1987). These correlations make it obvious that if a causative relationship exists between C-value and cell volume, then genome size could be subject to adaptive manipulation by natural selection acting on the organismal phenotype.

Proponents of the selfish and junk DNA hypotheses argue that the well-established and approximately linear relationship between DNA content and cell volume is strictly correlational; larger cells can simply tolerate more superfluous DNA. It is not clear, however, why the tolerance of cells scales in such a direct way with genome size that this relationship persists in a linear fashion across several orders of magnitude and among vastly diverse organisms with different replication requirements (Cavalier-Smith 1985b). Certainly, it is equally attractive to postulate a causal link between C-value and cell volume. Early attempts to describe such a link suggested that bulk DNA, by its sheer presence and independent of its nucleotide sequence, served to determine the volume of the cell by what came to be known as "nucleotypic" effects (Bennett 1972). Unfortunately, no mechanistic explanation for this causative relationship was offered. Later, CavalierSmith $(1978,1982,1985 b)$ argued that although cell volume was manipulated in response to natural selection, it was not determined by the amount of DNA present in the nucleus. Under this view, cell volume was modulated first via the actions of specific control genes, and genome sizes were then subsequently modified to meet the need for changes in the numbers of nuclear pores through which RNA could pass into the cytoplasm. Changes in the availability of such pores were believed to be accomplished by altering the volume of the nucleus, which, in turn, was determined by bulk DNA acting as a "nucleoskeleton."

Although genes capable of manipulating cell volume do exist (e.g., Nurse 1985), there is also strong evidence that noncoding DNA itself influences cell size. Changes in ploidy level, for example, have direct effects on cell volume. Although some authors have suggested that this is due to changes in regulatory gene dosage, the addition of noncoding supernumerary B chromosomes has a similar effect on cell size (Nurse 1985). Similarly, chromatin diminution (see below) leads to a reduction in cell volume (Cavalier-Smith 1980). Finally, the quantum patterns of genome size shifts described earlier provide a major challenge to the hypothesis that genome sizes are manipulated only secondarily in response to the nucleoskeletal needs of cells of different adaptively determined sizes. As Cavalier-Smith (1985b) pointed out, it is difficult to see why the nucleoskeletal requirements of related species should lead to the selection of genome sizes that are always multiples of some basal value. Again, it is more parsimonious to assume that bulk DNA content is capable, in concert with control genes, of influencing cell volumes, perhaps indirectly as a result of their nucleoskeletal effects on nuclear volume.

Under this assumption, it is clear that changes in genome size, via their influences on cell size, could be subject to strong selection pressures acting on the organismal phenotype. The potentially profound impacts of these effects on fitness are demonstrated persuasively by the strong relationship between cell size and morphological complexity in the brains of amphibians (Roth et al. 1994). In this case, even the modest changes in cell volume-and perhaps more importantly the delayed cellular developmental ratesassociated with increased genome size have clear effects on brain structure. Indeed, the simplified, almost paedomorphic appearance of the brains of amphibians with large genome sizes suggests strongly that had there been only a few more polyploidization events in our ancestry, humans would now be poorly equipped to cogitate the minutiae of genome size evolution. Similarly, it is relevant that birds (Hughes and Hughes 1995) and bats (Van Den Bussche et al. 1995), for which high metabolic rates are prerequisites for flight, have the smallest genomes among homeotherms, and that lungfishes, which must tolerate prolonged periods of estivation under hypoxic conditions where survival is aided by low metabolic rates, have the largest genomes of all vertebrates (Cavalier-Smith 1985a).

\section{Geographical Distribution of DNA Content Variation}

In addition to the cytological and physiological correlates discussed above, there exists a convincing correlation between DNA content and thermal regime. In diverse taxonomic groups there is a cline in DNA content with both latitude and altitude, with those species inhabiting northern (i.e., arctic) or high-altitude climates exhibiting larger genomes or more frequent polyploidy than their southern or low-altitude counterparts. For example, the freshwater fish fauna of arctic Canada is thoroughly dominated by salmonids, the only one of 20 fish families in North America consisting entirely of polyploids. Similarly, populations of the dominant zooplankton genera in arctic lakes, Daphnia and Bosmina, are typically composed of polyploids, whereas their temperate zone counterparts are diploid (Beaton and Hebert 1988). A similar climatedependent trend is apparent in Plethodon salamanders (Xia 1995; Jockusch 1997) and in numerous groups of plants (Grime and Mowforth 1982). It is obvious, therefore, that natural selection can-and does-favor the modulation of DNA content under certain condi- 
tions. This, coupled with the quantum nature of genome size shifts, suggests that there is a powerful and as-yet-undescribed mechanism(s) responsible for the adaptive manipulation of DNA content.

\section{Intraindividual Variation in DNA Content}

It has become common practice to neatly characterize organisms, or life stages of organisms, as haploid, diploid, or polyploid. In reality, the conglomerate of cells that makes up the body of an individual exists as a melange of ploidy levels; there is no such thing as a strictly diploid organism (Brodsky and Uryvaeva 1985). The DNA contents of cells within an individual organism are manipulated in two ways: The first involves the expansion of DNA content in somatic cells; the second results in the miniaturization of somatic genomes. The importance of both these processes in evolution has been sorely underestimated.

\section{Endopolyploidy and the Expansion of Somatic DNA}

Even more common than the occurrence of polyploidy among different species is the tendency of certain cells within organisms to exist in a polyploid state. This process of endopolyploidy has been observed in every animal species studied to date (including humans) and is especially common among arthropods. It is also prevalent in plants, particularly among angiosperms (Brodsky and Uryvaeva 1985), and in a few species nearly all tissues are endopolyploid (Galbraith et al. 1991). The mechanism by which this process occurs is well understood; endopolyploidy arises when mitotic DNA replication is not followed by cell division. In cases where the replicated chromosomes do not separate and remain juxtaposed, the cell is said to display polyteny rather than strict endopolyploidy, but the outcome is the same. Most biologists are aware of the presence of polytene chromosomes in the salivary glands of Drosophila, but fewer recognize that this is but one variant of a very common phenomenon.

Endoreduplication of DNA is obviously under genetic control as ploidy levels vary in a tissue-specific fashion. For example, in species of the crustacean Daphnia, nearly half of their DNA is in an endopolyploid state with ploidy levels varying among tissues from 2 to 2048C (Beaton and Hebert 1989). The modulation of ploidy levels is apparently mediated by natural selection favoring larger cell volumes, delayed cell cycles, or increased gene dosage in certain tissues. High levels of endopolyploidy are typical of cells involved in secretion or intense protein production, for example. In this regard, it is interesting to note that the highest level of endopolyploidy observed to date, exceeding one million-ploid, occurs in the silk-producing glands of the larval silkworm moth, Bombyx mori (PerdixGillot 1979), which has undergone intensive artificial selection over several thousand years to maximize silk production. It is also interesting to note that the highest levels of polyploidy are found in plants and animals with small basal genome sizes, suggesting that it results from an evolutionary compromise between selection pressures favoring the shorter generation times and rapid development afforded by small genomes (De Rocher et al. 1990) and those favoring the maintenance of a minimum mass of DNA in certain cells.

\section{Chromatin Diminution and the Deletion of Gametic DNA}

The second form of intraindividual DNA content modulation, chromatin diminution, occurs much less commonly than does endopolyploidy and has so far been identified in nematodes, copepods, ciliated protozoans, several dipteran families, and hagfish (Kubota et al. 1994). Chromatin diminution is a curious process whereby large amounts of DNA present in the zygote are deleted from early somatic cell lines at the 8-32 cell stage, resulting in gametic genomes that are substantially larger than expected based on inspection of somatic cells. Indeed, 2C somatic cells in Cyclops strenuus contain only $\sim 5 \%$ as much DNA as a newly fertilized egg (Grishanin et al. 1994). This phenomenon has traditionally been interpreted by proponents of selfish DNA as a process whereby invading sequences of parasitic DNA allow themselves to be deleted from the dead-end somatic cell lines, but not from the germ line. However, it is difficult to imagine how a collection of repetitive elements could exercise such a powerful influence over the genetic mechanisms of their "host."

A more complete understanding of the process of chromatin diminution may come from considering the differential modulation of gametic and somatic genomes as an adaptive feature that has arisen in response to nucleotypic selection. Many species that show chromatin diminution, including nematodes and some copepods, are known to exhibit determinate cell numbers. Thus, any large-scale manipulations of the somatic genome would have profound effects on adult body size via their influences on cell volumes. Thus, if there is selection for larger egg size, but selection against large body size - a situation that is not difficult to envision-then one would expect to find the evolution of mechanisms capable of either amplifying gametic and/or reducing somatic DNA contents. This could be accomplished by amplifying gametic amounts of noncoding DNA or by eliminating somatic satellite DNA and introns (Grishanin et al. 1996).

Alternatively, it may be useful to consider chromatin diminution and endopolyploidy as two sides of the same coin, rather than as unrelated phenomena. It is possible that the gametic nuclei of species that undergo chromatin diminution are highly polytenic, having undergone endoreduplication of the entire genome in response to nucleotypic selection. Hence, 
chromatin diminution may simply be the return of somatic cell lines to the diploid state via the deletion of these additional copies of the genome. This interpretation makes unnecessary any assumptions of complex intragenomic contests or fantastically precise pruning mechanisms and appeals only to the well-established phenomena of nucleotypic selection and endoreduplication. This hypothesis could be tested by examining the percentage of the genome represented by repetitive DNA before and after chromatin diminution. An unchanged proportion of noncoding DNA in the postdiminuted somatic genome would strongly suggest that entire copies of the genome, and not just select fragments, had been deleted during this process.

\section{Bridging (Some of) the Gaps in Punctuated Evolution}

The prevalence of quantum shifts in basal genome size and the ubiquity of intraindividual DNA content modulation make it clear that there exist simple mechanisms, presumably involving only one or a few control genes, that can effect large-scale changes in DNA content both within and among individual organisms. This obviously is of great importance in evolutionary biology, because it presents a plausible mechanism for the origin of large, rapid changes in key physiological and morphological parameters in response to selection acting on only a small number of genes. At last, it appears that at least one potential link between the gene-level view of natural selection espoused by population geneticists and the punctuated, organism-based interpretation of the fossil record advocated by palaeontologists has been found.

\section{Polyploidy and Diversification}

It has often been stated that polyploids have little evolutionary potential relative to their diploid counterparts because the multiplicity of their genomes buffers them against mutation. However, the high levels of heterozygosity found within most polyploids, the predominance of polyploidy in plant evolution, and the well-documented ability of polyploids to invade novel habitats (Lewis 1980) all suggest strongly that polyploidization presents no hindrance to evolutionary change. In addition, newly polyploid genomes are often highly unstable, resulting in a radical reshuffling of genes (Soltis and Soltis 1995; Song et al. 1995), which can result in rapid diversification. Polyploidy also results in nearly instantaneous speciation, because the altered chromosome complements of polyploid individuals typically lead to reproductive isolation from their diploid ancestors. Although this mode of speciation is much less common in animals than in plants, past polyploidization events lurk in the ancestry of most organisms, including vertebrates (Ohno et al. 1968; Comings 1972).

The duplication of single gene loci has long been considered a "prime factor of evolution" (Ohno et al. 1968). The redundancy of previously constrained coding regions and the multiplication of key genes involved in developmental regulation can have profound evolutionary implications (Ruddle 1997). Polyploids, which are configured with duplicates of all gene loci, often show bursts of evolutionary divergence following their origin (Iwake et al. 1996). For example, the multiplication of homeobox clusters following polyploidization may explain the enormous diversity of fish species (Amores et al. 1998; Vogel 1998).

\section{Genome Jumps and Novel Niches}

Aside from the effects arising through increased gene diversity, any increase in DNA content exerts nucleotypic effects. The cells of polyploid taxa are invariably larger than those of their diploid ancestors and so too are structures with determinate cell numbers. These nucleotypic effects undoubtedly also extend to taxa whose genome size shifts arose through cryptopolyploidy. These will include alterations in metabolic and developmental rates, somatic cell and gamete size, and, in cases where there is no compensatory change in cell numbers, overall body size. As such, a rapid change in DNA content can have major effects on the physiology and ecological lifestyle of an organism, impacting its ability to evade predators and acquire food as well as a host of other relevant aspects of its life history. Changes in genome size may not affect the "evolvability" of a lineage, but they may very well open up a new realm of ecological possibilities and selection pressures that lead to rapid change and speciation.

\section{The Hopeful Monster Gets Lucky}

The genome size distributions in many taxonomic assemblages make it clear that mutations involving quantum jumps in genome size and the associated changes in key organismal attributes have often been successful. This type of saltational change has often been dismissed on the grounds that such a mutant could not locate a mate that shared its newly acquired genetic and morphological characteristics; the hopeful monster would be without a monsterette. Thus, if a novel mutation is to persist, some mechanism must exist that improves the likelihood that two rare mutants can meet. In the case of DNA content modulation, this may be facilitated by the profound changes in developmental rates among such mutants that cause them to reach sexual maturity at a time offset from that of the remainder of the population.

\section{The Effects of Endopolyploidy}

Much like shifts in basal genome size, the occurrence of endopolyploidy sets the stage for abrupt changes in important physiological and morphological traits, and again can be mediated by the action of relatively few

\section{Genome Research}


control genes. In contrast to the phenotypic effects of basal genome size shifts, however, the impacts of these changes will be more localized, occurring only in certain tissues or cell types, and therefore involving changes in single characters. However, these characters often possess linkages to key fitness traits. For example, developing eggs of most arthropods originate from a tetrad of cells; one undergoes meiosis to become an egg, while the remainder differentiate into nurse cells that provision the egg with nutrients. In many species, these latter cells become endopolyploid, a process that enhances their ability to donate large volumes of essential nutrients. Not surprisingly, shifts in the ploidy levels of nurse cells can affect egg size. For example, in viviparous aphids, in which the eggs are nourished only briefly, the nurse cells reach a ploidy level of only 16C. In contrast, the nurse cells in oviparous females, which nourish the eggs for a prolonged period of time, rise to a ploidy level of 2048C (Manicardi et al. 1995). Endopolyploidy is also known to have morphological effects, as evidenced by the apparent link between the presence of endopolyploid cells and the formation of different predator-induced head morphologies in Daphnia (Beaton and Hebert 1997). If endopolyploidy can provoke morphological change within the lifespan of an individual, it can surely effect morphological change in the evolution of a lineage.

\section{Conclusions}

It is obvious that changes in DNA content both among and within individuals have profound effects on many phenotypic traits directly related to organismal fitness. The fact that genomic reconfigurations can occur in response to selection acting on only a few genes, yet provoke large-scale, rapid, phenotypic changes suggests that the neo-Darwinian paradigm can be considered neither dead nor complete. Specifically, an understanding of the structure, regulation, and evolutionary dynamics of genomes at large represents a necessary

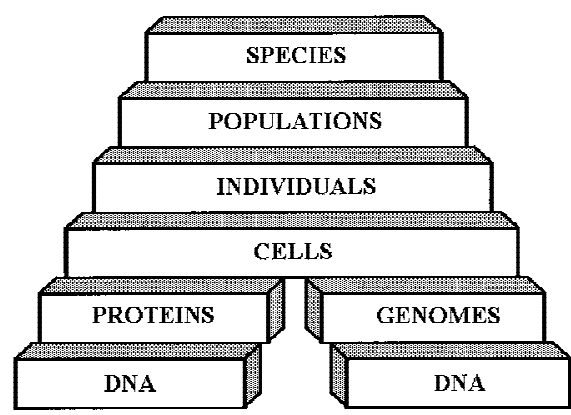

Figure 2 The revised "biological hierarchy," which recognizes the dual role of DNA in the evolutionary process. In addition to its protein-coding functions, bulk DNA exerts important effects on cellular parameters (independent of its specific nucleotide sequence), which can have profound fitness consequences at the organismal level. component of evolutionary theory that has been overlooked to date, as emphasized by its conspicuous omission from the traditional biological hierarchy. In reality, there exist separate but converging biological hierarchies (Fig. 2), with DNA playing a dual role-both in regard to its coding and regulatory functions, and in terms of its bulk effects independent of nucleotide sequence. It is only by acknowledging the multifaceted influence of DNA that a cohesive theory of evolution will be achieved.

\section{ACKNOWLEDGMENTS}

This work was supported by a Natural Sciences and Engineering Research Council of Canada (NSERC) grant to P.D.N.H. and NSERC postgraduate and University of Guelph Alumni Doctoral scholarships to T.R.G.

\section{REFERENCES}

Amores, A., A. Force, Y.-L. Yan, L. Joly, C. Amemiya, A. Fritz, R.K. Ho, J. Langeland, V. Prince, Y.-L. Wang, M. Westerfield, M. Ekker, and J.H. Postlethwait. 1998. Zebrafish hox clusters and vertebrate genome evolution. Science 282: 1711-1714.

Bachmann, K. 1972. Genome size in mammals. Chromosoma 37: 85-93.

Bachmann, K., B.A. Harrington, and J.B. Craig. 1972. Genome size in birds. Chromosoma 37: 405-416.

Beaton, M.J. and P.D.N. Hebert. 1988. Geographical parthenogenesis and polyploidy in Daphnia pulex. Am. Nat. 132: 157-163.

_ 1989. Miniature genomes and endopolyploidy in cladoceran crustaceans. Genome 32: 1048-1053.

- 1997. The cellular basis of divergent head morphologies in Daphnia. Limnol. Oceanogr. 42: 346-356.

Bennett, M.D. 1972. Nuclear DNA content and minimum generation time in herbaceous plants. Proc. R. Soc. Lond. B Biol. Sci. 181: 109-135.

Brodsky, V.Ya. and I.V. Uryvaeva. 1985. Genome multiplication in growth and development. Cambridge University Press, Cambridge, UK.

Cavalier-Smith, T. 1978. Nuclear volume control by nucleoskeletal DNA, selection for cell volume and cell growth rate, and the solution of the DNA C-value paradox. J. Cell Sci. 34: 247-278. . 1980. How selfish is DNA? Nature 285: 617-618.

- 1982. Skeletal DNA and the evolution of genome size. Annu. Rev. Biophys. Bioeng. 11: 273-302.

_. 1985a. Eukaryotic gene numbers, non-coding DNA, and genome size. In The evolution of genome size (ed. T. Cavalier-Smith), pp. 69-103. John Wiley \& Sons, Chichester, UK.

- 1985b. Cell volume and the evolution of eukaryote genome size. In The evolution of genome size (ed. T. Cavalier-Smith), pp. 105-184. John Wiley \& Sons, Chichester, UK.

Charlesworth, B. 1996. The changing sizes of genes. Nature 384: $315-316$.

Comings, D.E. 1972. Evidence for ancient tetraploidy and conservation of linkage groups in mammalian chromosomes. Nature 238: 455-457.

Dawkins, R. 1976. The selfish gene. Oxford University Press, Oxford, UK.

De Rocher, E.J., K.R. Harkins, D.W. Galbraith, and H.J. Bohnert. 1990. Developmentally regulated systemic endopolyploidy in succulents with small genomes. Science 250: 99-101.

Doolittle, W.F. and C. Sapienza. 1980. Selfish genes, the phenotype paradigm and genome evolution. Nature 284: 601-603.

Finston, T.L., P.D.N. Hebert, and R.B. Foottit. 1995. Genome size variation in aphids. Insect Biochem. Mol. Biol. 25: 189-196.

Galbraith, D.A., K.R. Harkins, and S. Knapp. 1991. Systemic 
endopolyploidy in Arabidopsis thaliana. Plant Physiol. 96: $985-989$.

Gambi, M.C., L. Ramella, G. Sella, P. Protto, and E. Aldieri. 1997. Variation in genome size of benthic polychaetes: Systemic and ecological relationships. J. Mar. Biol. Assoc. UK 77: 1045-1057.

Garagna, S., L. Rebecchi, and A. Guidi. 1996. Genome size variation in Tardigrada. Zool. J. Linn. Soc. 116: 115-121.

Gold, J.R., C.J. Ragland, and J.B. Woolley. 1992. Evolution of genome size in North American fishes. In Systematics, historical ecology, and North American freshwater fishes (ed. R.L. Mayden), pp. 534-550. Stanford University Press, Stanford, CA.

Grant, V. 1981. Plant speciation, 2nd ed. Columbia University Press, New York, NY.

Grime, J.P. and M.A. Mowforth. 1982. Variation in genome size-an ecological interpretation. Nature 299: 151-153.

Grishanin, A.K., V.Ya. Broskii, and A.P. Akif'ev. 1994. Somatic cells of Cyclops strenuus (Copepoda, Crustacea) lose about $90 \%$ of the genome during chromatin diminution. Dokl. Biol. Sci. 338: 505-506.

Grishanin, A.K., G.A. Khudolii, G.O. Shaikhaev, V.Ya. Brodskii, V.B. Makarov, and A.P. Akif'ev. 1996. Chromatin diminution in Cyclops kolensis (Copepoda, Crustacea) is a unique example of genetic engineering in nature. Russ. J. Genet. 32: 424-430.

Hawkins, J.D. 1988. A survey on intron and exon lengths. Nucleic Acids Res. 16: 9893-9908.

Hughes, A.L. and M.K. Hughes. 1995. Small genomes for better flyers. Nature 377: 391.

Hughes-Schrader, S. and F. Schrader. 1956. Polyteny as a factor in the chromosomal evolution of the Pentatomini (Hemiptera). Chromosoma 8: 135-151.

Iwake, N., K. Kuma, and T. Miyata. 1996. Evolution of gene families and relationship with organismal evolution: Rapid divergence of tissue-specific genes in the early evolution of chordates. Mol. Biol. Evol. 13: 483-493.

Jain, H.K. 1980. Incidental DNA. Nature 288: 647-648.

Jockusch, E.J. 1997. An evolutionary correlate of genome size change in plethodontid salamanders. Proc. R. Soc. Lond. B Biol. Sci. 264: 597-605.

John, B. and G.L.G. Miklos. 1988. The eukaryote genome in development and evolution, p. 11. Allen \& Unwin, London, UK.

Kersanach, R., H. Brinkmann, M.-F. Liaud, D.-X. Zhang, W. Martin, and R. Cerff. 1994. Five identical intron positions in ancient duplicated genes of eubacterial origin. Nature 367: 387-389.

Keyl, H.G. 1965. A demonstrable local and geometric increase in the chromosomal DNA of Chironomus. Experientia 21: 191-193.

Krawiec, S. and M. Riley. 1990. Organization of the bacterial chromosome. Microbiol. Rev. 54: 502-539.

Kubota, S., Y. Nakai, N. Sato, M. Kuro-o, and S. Kohno. 1994. Chromosome elimination in Northeast Pacific hagfish, Eptatretus stoutii (Cyclostomata, Agnatha). J. Hered. 85: 413-415.

Lewis, W.H. 1980. Polyploidy: Biological relevance. Plenum Press, New York, NY.

Li, W.-H. 1997. Molecular evolution. Sinauer Associates, Inc., Sunderland, MA.

Manicardi, C.C., E. Galli, A. Malavasi, and A.M. Bonvinci Pogliai. 1995. DNA content in the nurse cell nuclei of viviparous and oviparous females of Megoura viciae (Homoptera, Aphididae). Invert. Reprod. Develop. 28: 1-6.

Maynard Smith, J. 1982. Overview-unsolved evolutionary problems. In Genome evolution (ed. G.A. Dover and R.B. Flavell), pp.375-382. Academic Press, New York, NY.

McLaren, I.A., J.-M. Sévigny, and B.W. Frost. 1989. Evolutionary and ecological significance of genome sizes in the copepod genus Pseudocalanus. Can. J. Zool. 67: 565-569.

Mirsky, A.E. and H. Ris. 1951. The deoxyriboneucleic acid content of animal cells and its evolutionary significance. J. Genet. Physiol. 34: $451-462$.

Moriyama, E.N., D.A. Petrov, and D.L. Hartl. 1998. Genome size and intron size in Drosophila. Mol. Biol. Evol. 15: 770-773.
Narayan, R.K.J. 1982. Discontinuous DNA variation in the evolution of plant species: The genus Lathyrus. Evolution 36: 877-891.

- 1983. DNA distribution in the chromosomes of Lathyrus species. Genetica 61: 47-53.

Nurse, P. 1985. The genetic control of cell volume. In The evolution of genome size (ed. T. Cavalier-Smith), pp. 185-196. John Wiley \& Sons, Chichester, UK.

Ohno, S. 1972. So much "junk" DNA in our genome. In Evolution of genetic systems (ed. H.H. Smith), pp. 366-370. Gordon and Breach, New York.

Ohno, S., U. Wolf, and N.B. Atkin. 1968. Evolution from fish to mammals by gene duplication. Hereditas 59: 169-187.

Orgel, L.E. and F.H.C. Crick. 1980. Selfish DNA: The ultimate parasite. Nature 284: 604-607.

Pagel, M. and R.A. Johnstone. 1992. Variation across species in the size of the nuclear genome supports the junk-DNA explanation for the C-value paradox. Proc. R. Soc. Lond. B Biol. Sci. 249: 119-124.

Perdix-Gillot, S. 1979. DNA synthesis and endomitosis in the giant nuclei of the silkgland of Bombyx mori. Biochemie 61: 171-204.

Petrov, D.A. and D.L. Hartl. 1997. Trash DNA is what gets thrown away: High rate of DNA loss in Drosophila. Gene 205: 279-289.

Petrov, D.A., E.R. Lozovskaya, and D.L. Hartl. 1996. High intrinsic rates of DNA loss in Drosophila. Nature 384: 346-349.

Rees, H. 1972. DNA in higher plants. In Evolution of genetic systems (ed. H.H. Smith), pp. 394-418. Gordon and Breach, New York, NY.

Roth, G., J. Blanke, and D.B. Wake. 1994. Cell size predicts morphological complexity in the brains of frogs and salamanders. Proc. Natl. Acad. Sci. 91: 4796-4800.

Rothfels, K., E. Sexsmith, M. Heimburger, and M.O. Krause. 1966. Chromosome size and DNA content of species of Anemone L. and related genera (Ranunculaceae). Chromosoma 20: 54-74.

Ruddle, F.H. 1997. Vertebrate genome evolution-the decade ahead. Genomics 46: 171-173.

Russell, C.B., D. Fraga, and R.D. Hinrichsen. 1994. Extremely short 20-33 nucleotide introns are the standard length in Paramecium tetraurelia. Nucleic Acids Res. 22: 1221-1225.

Sella, G., C.A. Redi, L. Ramella, R. Soldi, and M.C. Premoli. 1993. Genome size and karyotype in some interstitial polychaete species of the genus Ophryotrocha (Dorvilleidae). Genome 36: $652-657$.

Sessions, S.K. and A. Larson. 1987. Developmental correlates of genome size in plethodontid salamanders and their implications for genome evolution. Evolution 41: 1239-1251.

Soltis, D.E. and P.S. Soltis. 1995. The dynamic nature of polyploid genomes. Proc. Natl. Acad. Sci. 92: 8089-8091.

Song, K., P. Lu., and T.C. Osborn. 1995. Rapid genomic change in synthetic polyploids of Brassica and its implications for polyploid evolution. Proc. Natl. Acad. Sci. 92: 7719-7723.

Sparrow, A.H. and A.F. Naumann. 1976. Evolution of genome size by DNA doublings. Science 192: 524-527.

Thomas, C.A. 1971. The genetic organization of chromosomes. Annu. Rev. Genet. 5: 237-256.

Van Den Bussche, R.A., J.L. Longmire, and R.J. Baker. 1995. How bats achieve a small C-value: frequency of repetitive DNA in Macrotus. Mamm. Genome 6: 521-525.

Vinogrodov, A.E. 1995. Nucleotypic effect in homeotherms: Body mass-corrected basal metabolic rate of mammals is related to genome size. Evolution 49: 1249-1259.

- 1997. Nucleotypic effect in homeotherms: Body-mass independent resting metabolic rate of passerine birds is related to genome size. Evolution 51: 220-225.

Vogel, G. 1998. Doubled genes may explain fish diversity. Science 281: $1119-1120$.

Wallace, D.C. and H.J. Morowitz. 1973. Genome size and evolution. Chromosoma 40: 121-126.

Xia, X. 1995. Body temperature, rate of biosynthesis, and evolution of genome size. Mol. Biol. Evol. 12: 834-842. 


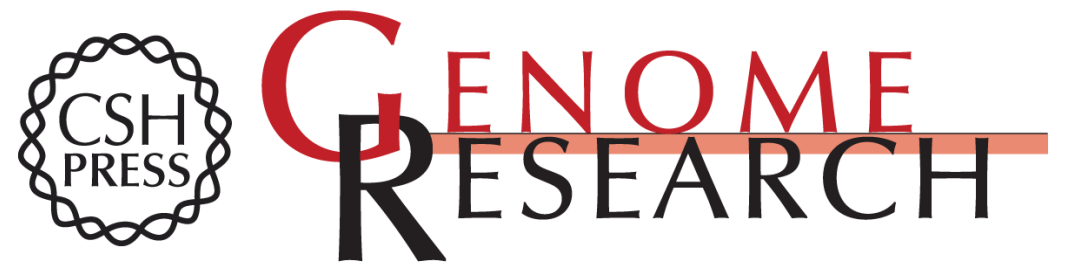

\section{The Modulation of DNA Content: Proximate Causes and Ultimate Consequences}

T. Ryan Gregory and Paul D.N. Hebert

Genome Res. 1999 9: 317-324

Access the most recent version at doi:10.1101/gr.9.4.317

References This article cites 54 articles, 10 of which can be accessed free at:

http://genome.cshlp.org/content/9/4/317.full.html\#ref-list-1

\section{License}

Email Alerting Receive free email alerts when new articles cite this article - sign up in the box at the Service top right corner of the article or click here.

\section{Affordable, Accurate Sequencing.}

To subscribe to Genome Research go to: https://genome.cshlp.org/subscriptions 